\title{
RECUPERAÇÃO DA PRODUTIVIDADE DE SOQUEIRAS DE CANA DE AÇÚCAR COM ADUBAÇÃO NPK
}

\author{
RATON SUGAR CANE PRODUCTIVITY RECOVERING \\ WITH NPK FERTILIZATION
}

\author{
Heroldo WEBER ${ }^{1}$ \\ Edelclaiton DAROS ${ }^{2}$ \\ José Luiz Camargo. ZAMBON ${ }^{2}$ \\ Oswaldo Teruyo. IDO ${ }^{3}$ \\ José Donisete BARELA ${ }^{4}$
}

\begin{abstract}
RESUMO
Com o objetivo de avaliar a influência da adubação sobre a produtividade de soqueiras de cana-de-açúcar, que não haviam sido adubadas em colheitas anteriores, foram conduzidos dois experimentos no campo, em Latossolo Roxo, na Usina Casquel, Cambará, PR. O delineamento experimental foi o de blocos casualizados, com cinco repetições constando da aplicação de oito tratamentos em parcelas de cinco sulcos de $15 \mathrm{~m}$ de comprimento e espaçamento entre linhas de $1,3 \mathrm{~m}$. Os tratamentos foram: o $\mathrm{N}$ nas doses de $0,50,100$ e $150 \mathrm{~kg}^{-h^{-1}}$; a associação da dose de $100 \mathrm{~kg} \cdot \mathrm{ha}^{-1}$ de N com as de 50, 100 e $150 \mathrm{~kg} \cdot \mathrm{ha}^{-1}$ de $\mathrm{K}_{2} \mathrm{O}$; e a associação da dose de $100 \mathrm{~kg} \cdot \mathrm{ha}^{-1}$ de N com a de $100 \mathrm{~kg} \cdot \mathrm{ha}^{-1}$ de $\mathrm{K}_{2} \mathrm{O}$ e com a de $100 \mathrm{~kg} \cdot \mathrm{ha}^{-1}$ de $\mathrm{P}_{2} \mathrm{O}_{5}$. Os resultados obtidos em canaviais de terceira soqueira mostraram que a recuperação da produtividade agrícola foi da ordem de $48,5 \%$ e $34,4 \%$ com a aplicação da adubação recomendada contendo $\mathrm{N}$ e K, respectivamente, para as áreas experimentais onde os tratamentos foram aplicados aos 70 e 90 dias após o corte. A reaplicação dos tratamentos, em quarta soqueira, quarenta e cinco dias após a primeira colheita de um dos experimentos apresentou, no ano seguinte, acréscimos na produtividade de até $76,2 \%$.
\end{abstract}

Palavras-chave: cana-soca, adubação nitrogenada, adubação potássica.

\begin{abstract}
With the objective of evaluating the influence of the fertilization on the raton productivity that had not been fertilized in the previous courts, two trials were done to field, in soil Latossolo Roxo, in Usina Casquel, Cambará, PR. The experimental design was randomized complete blocks, with five replications to application of eight treatments, with experimental units of 5 rows of $15 \mathrm{~m}$ of length and spacing among rows of $1.3 \mathrm{~m}$. The treatments were only $N\left(0,50,100\right.$ and $\left.150 \mathrm{~kg} \cdot \mathrm{ha}^{-1}\right)$, the association of $N$ and $K\left(100 \mathrm{~kg} \cdot \mathrm{ha} \mathrm{a}^{-1} \mathrm{~N}\right.$ with 50,100 and $\left.150 \mathrm{~kg} \cdot \mathrm{ha}^{-1} \mathrm{~K}_{2} \mathrm{O}\right)$ and the association of NPK $\left(100 \mathrm{~kg} \cdot \mathrm{ha}^{-1} \mathrm{~N}\right.$ $\left.+100 \mathrm{~kg} \cdot \mathrm{ha}^{-1} \mathrm{~K}_{2} \mathrm{O}+100 \mathrm{~kg} \cdot \mathrm{ha}^{-1} \mathrm{P}_{2} \mathrm{O}_{5}\right)$. The results obtained in sugar cane of 3rd raton, show that the recovery of the agricultural productivity reached yields of $48.5 \%$ and $34.4 \%$ with the application of the recommended fertilization, having $N$ and $K$, respectively, for the areas where the treatments were applied to the 70 and 90 days after harvesting. In one of trials the reapplication of the treatments, in $4^{\text {th }}$ ratoon, 45 days after harvesting, presented increments in the productivity of up to $76.2 \%$.
\end{abstract}

Key words: raton sugarcane, nitrogen fertilization, potassium fertilization.

\footnotetext{
1 Engenheiro Agrônomo, Doutor, Universidade Federal do Paraná, Departamento de Fitotecnia e Fitossanitarismo, Rua dos Funcionários, 1540, CEP 80035-050, Curitiba, PR. $=-$ Autor para correspondência.

2 Engenheiro Agrônomo, Doutor, Universidade Federal do Paraná, Departamento de Fitotecnia e Fitossanitarismo, Professor Adjunto, Rua dos Funcionários, 1540, CEP 80035-020, Curitiba, PR.

${ }^{3}$ Engenheiro Agrônomo, Mestre, Universidade Federal do Paraná, Departamento de Fitotecnia e Fitossanitarismo, Professor Assistente, Rua dos Funcionários, 1540, CEP 80035-020, Curitiba, PR.

${ }^{4}$ Engenheiro Agrônomo, Usina Casquel, Cambará, PR.
} 


\section{INTRODUÇÃO}

A cana-de-açúcar para produção de álcool e açúcar é cultivada, em média, por quatro a cinco cortes. Assim os ciclos da cana-soca podem representar até $90 \%$ da área plantada. Apesar dessa representatividade, recomendações de adubação para soqueiras têm sido pouco estudadas, sobretudo tendo em vista que atualmente se pretende aumentar a eficiência e diminuir custos do sistema de produção dessa cultura.

Na década 1990 foram escassos os trabalhos que tiveram por objetivo avaliar a reação das soqueiras à adubação. Informações nesse sentido tiveram maior divulgação nas décadas de 1970 e 1980. Para a maioria das regiões canavieiras do Brasil, Marinho (1974) [6], em Alagoas, Santos et al. (1979) [10], em Pernambuco e Paraíba, Azeredo et al. (1984) [2], no Espírito Santo, Azeredo et al. (1980) [1], em Minas Gerais, e Zambello Júnior e Azeredo (1983) [14], em São Paulo, relatam que nos ciclos da cana-soca a resposta à adubação nitrogenada é maior que para a cana-planta. De acordo com Zambello Júnior e Azeredo (1983) [14] a reação da cana-soca ao potássio, normalmente, é da mesma ordem de grandeza da observada para a cana-planta. Em solos com baixos teores de fósforo, Bolsanello et al. (1993) [3] indicam que a aplicação da dose recomendada de fósforo no plantio é suficiente para suprir as necessidades da cana-deaçúcar até, pelo menos, quatro cortes.

Com o preço em baixa e a produção e os estoques em alta, favorecidos pelo ano safra 1998/99 quanto a precipitações fartas e bem distribuídas, entre as ações de racionalização de gastos está, entre outras, a redução nos tratos culturais, particularmente a adubação de soqueiras, que representa quatro quintos da área total cultivada
(Nunes Júnior, 1999) [7]. Embora esse autor cite que a redução da adubação tenha atingido, em 1999 , cerca de $37 \%$ das unidades produtoras de açúcar e álcool do Estado de São Paulo, tem-se a informação de que essa redução ocorreu em várias regiões produtoras de cana do País.

Assim, esse trabalho foi conduzido com o objetivo de avaliar a resposta da cana-de-açúcar, em estádio de terceira soqueira não adubada anteriormente, à aplicação dos nutrientes recomendados para esse ciclo, buscando-se por esse meio a sua recuperação e, conseqüentemente, aumento na sua longevidade.

\section{METODOLOGIA}

Os experimentos foram conduzidos em área comercial de cana-de-açúcar, em um Latossolo Roxo cultivado com a variedade RB72454, na Usina Casquel, Cambará, PR, no sistema de colheita manual com queima. O primeiro foi instalado na Fazenda Fonte Clara, em área colhida em 04/10/1997. Nessa área os tratamentos foram aplicados em 18/12/1997 e a colheita foi feita após dez meses, com a cana-de-açúcar em estágio de terceira soqueira (quarto corte), não adubada anteriormente. Nesse experimento os tratamentos foram reaplicados em 02/11/1998, 45 dias após a colheita, e a quarta soqueira (quinto corte) foi colhida novamente dez meses depois. $O$ segundo experimento foi instalado na Fazenda Água Suja, em área colhida em 14/10/1997. Os tratamentos foram aplicados em 13/01/1998 e a colheita da cana foi feita 11 meses depois, em estágio de terceira soqueira, não adubada anteriormente. A análise do solo revelou os valores que são apresentados no Quadro 1.

QUADRO 1 - Resultados das análises químicas dos solos dos experimentos, Usina Casquel, Cambará, PR, $1997 / 98$

\begin{tabular}{|c|c|c|c|c|c|c|}
\hline \multirow{2}{*}{ Local } & \multirow{2}{*}{$\begin{array}{c}\mathrm{pH} \\
\left(\mathrm{H}_{2} \mathrm{O}\right)\end{array}$} & $\mathrm{P}$ & $\mathrm{K}$ & $\mathrm{Ca}$ & $\mathrm{Mg}$ & $\mathrm{Al}$ \\
\hline & & $\left(\mathrm{mg} \cdot \mathrm{dm}^{-3}\right)$ & \multicolumn{4}{|c|}{ - $\left(\mathrm{cmol}_{\mathrm{c}} \cdot \mathrm{dm}^{-3}\right)^{-}$} \\
\hline Fazenda Fonte Clara & 5,0 & 4,0 & 0,09 & 4,9 & 1,8 & 0 \\
\hline Fazenda Água Suja & 5,3 & 4,0 & 0,11 & 8,0 & 3,3 & 0 \\
\hline
\end{tabular}

O delineamento experimental foi o de blocos casualizados, com cinco repetições. As parcelas com cinco linhas de $15 \mathrm{~m}$ de comprimento e espaçadas de $1,3 \mathrm{~m}$. Os oito tratamentos estão apresentados no Quadro 2 e foram aplicados lateralmente à linha da cana $(15 \mathrm{~cm})$ e incorporados com enxada.

As três linhas centrais de cada parcela foram utilizadas para a avaliação do rendimento agrícola $\left(\mathrm{t} . \mathrm{h}^{-1}\right)$, número médio de colmos por metro de sulco e massa média de um colmo.

Os resultados das avaliações foram submetidos à análise de variância empregando-se o programa MSTAT-C. As variáveis se mostraram homogêneas pelo teste de Bartlett e quando os tratamentos apresentaram significância a $5 \%$ de probabilidades, as médias foram comparadas pelo teste Duncan, no mesmo nível.

\section{RESULTADOS E DISCUSSÃO}

Os resultados apresentados na Tabela 1 revelam, para o experimento conduzido em terceira soqueira, na Fazenda Fonte Clara, que as maiores produtividades foram alcançadas com a aplicação de $100 \mathrm{~kg} \cdot \mathrm{ha}^{-1}$ de N e $100 \mathrm{~kg} \cdot \mathrm{ha}^{-1}$ de $\mathrm{K}_{2} \mathrm{O}$ e $100 \mathrm{~kg} \cdot \mathrm{ha}^{-1}$ de $\mathrm{P}_{2} \mathrm{O}_{5}$, com ganho de $53,9 \%$ na produtividade. 
QUADRO 2 - Tratamentos dos experimentos conduzidos na Usina Casquel, Cambará, PR, 199798

\begin{tabular}{cccc}
\hline \multirow{2}{*}{ Tratamento } & $\mathrm{N}$ & $\mathrm{K}_{2} \mathrm{O}$ & $\mathrm{P}_{2} \mathrm{O}_{5}$ \\
\cline { 2 - 4 } & \multicolumn{3}{c}{$\mathrm{kg} \cdot \mathrm{ha}^{-1}$} \\
\hline 1 & 0 & 0 & 0 \\
2 & 50 & 0 & 0 \\
3 & 100 & 0 & 0 \\
4 & 150 & 0 & 0 \\
5 & 100 & 50 & 0 \\
6 & 100 & 100 & 0 \\
7 & 100 & 150 & 0 \\
8 & 100 & 100 & 100 \\
\hline
\end{tabular}

A redução da dose aplicada, até a retirada total dos nutrientes $\mathrm{N}, \mathrm{P}$ e K da adubação, provocou decréscimo no peso médio dos colmos. Isso refletiu na produtividade final de colmos, em função da baixa disponibilidade desses nutrientes para o ciclo da cana-soca.

De acordo com Nunes Júnior (1999) [7] a redução média esperada de produtividade na ausência da adubação está em torno de $30 \%$ para solos de boa e média fertilidade. Entretanto, os canaviais que não recebem qualquer quantidade de adubo não formam boa quantidade de rizomas. Isso pode comprometer a produtividade das soqueiras seguintes, mesmo considerando que esses canaviais voltem a receber adubação normalmente.

TABELA 1 - Número médio de colmos por metro de sulco, massa média unitária de colmos e rendimento colmos, em terceira soqueira, Fazenda Fonte Clara, Cambará, PR. 1997/98 ${ }^{1}$

\begin{tabular}{|c|c|c|c|c|c|c|}
\hline \multicolumn{3}{|c|}{ Tratamentos $\left(\mathrm{kg} \cdot \mathrm{ha}^{-1}\right)$} & \multicolumn{4}{|c|}{ Colmos } \\
\hline $\mathrm{N}$ & $\mathrm{K}_{2} \mathrm{O}$ & $\mathrm{P}_{2} \mathrm{O}_{5}$ & $\begin{array}{c}\text { Número por } \\
\text { metro }\end{array}$ & $\begin{array}{c}\text { Massa Unitária } \\
\text { (g) }\end{array}$ & $\begin{array}{c}\text { Rendimento } \\
\left(\mathrm{t} \mathrm{ha}^{-1}\right)\end{array}$ & $\begin{array}{c}\text { Ganho } \\
(\%)\end{array}$ \\
\hline 0 & 0 & 0 & $9,2 \mathrm{~b}$ & $804 \mathrm{~d}$ & $56,41 \mathrm{~d}$ & 0,0 \\
\hline 50 & 0 & 0 & $10,1 \mathrm{ab}$ & $900 \mathrm{~cd}$ & $69,23 \mathrm{c}$ & 22,7 \\
\hline 100 & 0 & 0 & $9,8 a b$ & $1.027 \mathrm{abc}$ & $76,75 \mathrm{bc}$ & 36,0 \\
\hline 150 & 0 & 0 & $10,0 a b$ & 955 bc & $74,01 \mathrm{c}$ & 31,2 \\
\hline 100 & 50 & 0 & $10,3 \mathrm{a}$ & $1.057 \mathrm{ab}$ & $83,76 a b$ & 48,5 \\
\hline 100 & 100 & 0 & $9,7 a b$ & $1.134 \mathrm{a}$ & $84,10 a b$ & 48,5 \\
\hline 100 & 150 & 0 & $9,9 a b$ & $1.019 a b c$ & $77,43 a b c$ & 37,2 \\
\hline 100 & 100 & 100 & $10,1 \mathrm{ab}$ & $1.119 \mathrm{a}$ & $86,83 \mathrm{a}$ & 53,9 \\
\hline \multicolumn{3}{|c|}{ Coeficiente de Variação (\%) } & 8,55 & 10,88 & 10,81 & \\
\hline
\end{tabular}

1 Médias seguidas das mesmas letras não diferem a $5 \%$ de probabilidade pelo Teste de Duncan

$\mathrm{Na}$ Tabela 2 encontram-se os resultados obtidos no experimento instalado na Fazenda Fonte Clara, onde os tratamentos foram reaplicados em quarta soqueira após a colheita. Esses resultados revelam que a reaplicação dos tratamentos acentuou o efeito negativo da retirada da adubação na produtividade, podendo-se observar que o efeito dos tratamentos se fez presente tanto no número de colmos por metro de sulco quanto no peso unitário dos colmos, refletindo diretamente sobre a produtividade final de colmos.

Esses resultados também mostram que a adubação potássica, na quarta soqueira, em complemento à adubação nitrogenada, proporcionou aumentos de produtividade que chegaram a $76,2 \%$ em relação à testemunha e a mais de $50 \%$, quando comparada com a produtividade dos tratamentos que só receberam o adubo nitrogenado.

Em Latossolo Roxo, no Estado de São Paulo, Orlando Filho (1978) [8] verificou que uma tonelada de colmos de cana-soca requereu $0,68 \mathrm{~kg}$ de nitrogênio. Raij et al. (1996) [9] recomendam que a aplicação de nitrogênio em cana-soca seja baseada na produtividade esperada, indicando que se aplique um quilograma de $\mathrm{N}$ por tonelada estimada de colmos.

O efeito menos acentuado sobre o número de colmos por metro de sulco, Tabelas 1 e 3 , pode estar relacionado à época da aplicação dos tratamentos, aproximadamente 70 e 90 dias após o corte, respectivamente, estando as soqueiras já perfilhadas, enquanto que, os resultados da Tabela 2 apresentam aumento no número médio de colmos por metro de sulco, sendo que a reaplicação dos tratamentos foi feita 45 dias após a colheita. Humbert (1974) [5] cita que a aplicação de nitrogênio, normalmente, resulta em aumento na produção de perfilhos novos.

Mesmo aplicados tardiamente nitrogênio, fósforo e potássio teriam grande importância, pois de acordo com Golden (1961) [4] as soqueiras de canade-açúcar, após a décima semana de idade, absorvem $77 \%$ do nitrogênio, $85 \%$ do fósforo e $84 \%$ do potássio,. Embora não tenham contribuído no desenvolvimento inicial, logo após o corte, quando a cana-soca apresenta grande demanda desses nutrientes para o seu estabelecimento.

$\mathrm{Na}$ Tabela 3 estão os resultados que foram obtidos na Fazenda Água Suja e mostram a mesma tendência de aumento de peso médio de colmos e de produtividade verificado no experimento anterior (Tabelas 1 e 2), entretanto, observa-se que as margens de ganho, nesse segundo experimento, apresentam-se mais estreitas sugerindo que a aplicação dos tratamentos aos 90 dias pode ter sido muito tardia. 
TABELA 2 - Número médio de colmos por metro de sulco, massa média unitária de colmos e rendimento de colmos, em quarta soqueira, Fazenda Fonte Clara, Cambará, PR, 1997/98 ${ }^{1}$

\begin{tabular}{|c|c|c|c|c|c|c|}
\hline \multicolumn{3}{|c|}{ Tratamentos $\left(\mathrm{kg} \cdot \mathrm{ha}^{-1}\right)$} & \multicolumn{4}{|c|}{ Colmos } \\
\hline $\mathrm{N}$ & $\mathrm{K}_{2} \mathrm{O}$ & $\mathrm{P}_{2} \mathrm{O}_{5}$ & $\begin{array}{c}\text { Número por } \\
\text { metro }\end{array}$ & $\begin{array}{c}\text { Massa Unitária } \\
\text { (g) }\end{array}$ & $\begin{array}{c}\text { Rendimento } \\
\left(\mathrm{t} \mathrm{ha}^{-1}\right)\end{array}$ & $\begin{array}{c}\text { Ganho } \\
(\%)\end{array}$ \\
\hline 0 & 0 & 0 & $7,8 \mathrm{~d}$ & $896 \mathrm{c}$ & $53,93 \mathrm{~d}$ & 0,0 \\
\hline 50 & 0 & 0 & $8,1 \mathrm{~cd}$ & $900 \mathrm{c}$ & $57,23 \mathrm{~cd}$ & 6,0 \\
\hline 100 & 0 & 0 & 8,8 bc & $970 \mathrm{c}$ & 65,84 c & 22,0 \\
\hline 150 & 0 & 0 & $8,3 \mathrm{~cd}$ & 977 c & $62,93 \mathrm{~cd}$ & 16,7 \\
\hline 100 & 50 & 0 & 9,8 a & $1.107 \mathrm{~b}$ & 83,97 b & 55,7 \\
\hline 100 & 100 & 0 & $9,3 a b$ & $1.193 a b$ & $84,62 a b$ & 56,9 \\
\hline 100 & 150 & 0 & 9,8 a & $1.262 \mathrm{a}$ & 95,03 a & 76,2 \\
\hline 100 & 100 & 100 & $9,5 a b$ & $1.245 \mathrm{a}$ & $91,66 a b$ & 69,9 \\
\hline \multicolumn{3}{|c|}{ Coeficiente de Variação (\%) } & 8,55 & 10,50 & 12,45 & \\
\hline & & & $\mathrm{rde} \mathrm{K} \mathrm{no} \mathrm{so}$ & $0,09 \mathrm{cmol}_{\mathrm{c}} \cdot \mathrm{dm}^{-3}$ & & \\
\hline
\end{tabular}

1 Médias seguidas das mesmas letras não diferem a $5 \%$ de probabilidade pelo Teste de Duncan

TABELA 3 - Número médio de colmos por metro de sulco, massa média unitária de colmos e rendimento de colmos, em terceira soqueira, Fazenda Água Suja, Cambará, PR, 1997/98 ${ }^{1}$

\begin{tabular}{|c|c|c|c|c|c|c|}
\hline \multicolumn{3}{|c|}{ Tratamentos (kg.ha $\left.{ }^{-1}\right)$} & \multicolumn{4}{|c|}{ Colmos } \\
\hline $\mathrm{N}$ & $\mathrm{K}_{2} \mathrm{O}$ & $\mathrm{P}_{2} \mathrm{O}_{5}$ & $\begin{array}{l}\text { Número por } \\
\text { metro }\end{array}$ & $\begin{array}{l}\text { Massa Unitária } \\
\text { (a) }\end{array}$ & $\begin{array}{l}\text { Rendimento } \\
\left(\mathrm{t} \mathrm{ha}^{-1}\right)\end{array}$ & $\begin{array}{c}\text { Ganho } \\
(\%)\end{array}$ \\
\hline 0 & 0 & 0 & $9,8 a b$ & $876 \mathrm{~b}$ & $65,63 d$ & 0,0 \\
\hline 50 & 0 & 0 & $10,4 a$ & $960 \mathrm{~b}$ & $76,57 \mathrm{bc}$ & 16,7 \\
\hline 100 & 0 & 0 & $10,6 a$ & $919 \mathrm{~b}$ & $73,83 \mathrm{~cd}$ & 12,5 \\
\hline 150 & 0 & 0 & $10,6 a$ & $879 b$ & $71,61 \mathrm{~cd}$ & 9,1 \\
\hline 100 & 50 & 0 & $8,2 \mathrm{~b}$ & $1.080 \mathrm{a}$ & $73,83 \mathrm{~cd}$ & 12,5 \\
\hline 100 & 100 & 0 & $10,4 a$ & $1.195 \mathrm{a}$ & $85,46 a b$ & 30,0 \\
\hline 100 & 150 & 0 & $10,7 \mathrm{a}$ & $1.069 \mathrm{a}$ & 88,19 a & 34,4 \\
\hline 100 & 100 & 100 & $11,2 \mathrm{a}$ & $1.064 \mathrm{a}$ & $91,61 \mathrm{a}$ & 39,6 \\
\hline \multirow{2}{*}{\multicolumn{3}{|c|}{ Coeficiente de Variação (\%) }} & 12,39 & 7,71 & 11,42 & \\
\hline & & & \multicolumn{4}{|c|}{ Teor de $\mathrm{K}$ no solo: $0,11 \mathrm{cmol}_{\mathrm{C}} \cdot \mathrm{dm}^{-3}$} \\
\hline
\end{tabular}

1 Médias seguidas das mesmas letras não diferem a $5 \%$ de probabilidade pelo Teste de Duncan

Apesar da resposta à aplicação do $\mathrm{N}$, verificase que, nas duas áreas experimentais, a complementação com potássio apresentou efeito significativamente positivo no aumento da produtividade.

Os resultados obtidos por Weber e Azeredo (1997) [12] revelam que para a região canavieira do Estado do Espírito Santo as doses de $80 \mathrm{~kg} \cdot \mathrm{ha}^{-1}$ de $\mathrm{N}$ e de $80 \mathrm{~kg} \cdot \mathrm{ha}^{-1}$ de $\mathrm{K}_{2} \mathrm{O}$ resultaram em aumentos da ordem de $21 \%$ na produtividade das soqueiras.

Para os resultados apresentados nas Tabelas 1 e 3 observa-se um ganho na produção de colmos nas parcelas que receberam fósforo em cobertura, incorporado lateralmente à linha de cana juntamente com o nitrogênio e com o potássio. O ganho com a aplicação dos três nutrientes foi de $53,9 \%$ e $39,6 \%$ quando comparado às produtividades das testemunhas, sem adubação, e de $4,9 \%$ e $5,2 \%$ quando comparado aos tratamentos que receberam $\mathrm{N}$ e $\mathrm{K}$, respectivamente, nas Fazendas Fonte Clara (Tabela 1) e Água Suja (Tabela 3). Como a cultura não havia sido adubada anteriormente, observa-se pequena recuperação das soqueiras com a aplicação do fósforo tardiamente, em terceira soqueira. De acordo com Bolsanello et al. (1993) [3] quando a aplicação do fósforo é feita corretamente, por ocasião do plantio, ele é suficiente para atender, pelo menos, a quatro cortes e a aplicação tardia desse nutriente poderá aumentar a produtividade, mas não irá recuperar o potencial produtivo da cultura.

A dose de $100 \mathrm{~kg} \cdot \mathrm{ha}^{-1}$ de $\mathrm{P}_{2} \mathrm{O}_{5}$, aplicada nesses experimentos, foi decorrente das fórmulas disponíveis por ocasião da instalação dos mesmos. Para Raij et al. (1996) [9] a dose de $\mathrm{P}_{2} \mathrm{O}_{5}$ recomendada para a cana-soca é de $30 \mathrm{~kg} \cdot \mathrm{ha}^{-1}$, em canaviais adubados no plantio, quando $P$ (resina) for menor que $15 \mathrm{mg} . \mathrm{dm}^{-3}$.

Apesar de não ter sido possível fazer a determinação das características tecnológicas das canas colhidas nas áreas experimentais, de acordo com a literatura, se forem aplicadas as doses recomendadas de nitrogênio, fósforo e potássio para as soqueiras, existe a probabilidade de haver pouca ou nenhuma interferência na qualidade industrial da cultura, conforme trabalhos, entre outros, os conduzidos por Silva et al. (1977) [11] e Zambello Júnior et al. (1977) [13]. Ainda, segundo os mesmos autores, a variação na produção de açúcar, quando ocorre, é devida aos efeitos positivos da adubação sobre a produtividade de colmos. 
Conforme apresentado anteriormente a redução e/ou a omissão das doses e de nutrientes recomendados para a adubação de soqueiras de cana-de-açúcar deve ser uma decisão baseada em critérios e observações locais, pois os resultados permitem o planejamento dos cortes na adubação recomendada ou a manutenção da mesma em áreas estratégicas de alta produtividade. Os resultados demonstram que o manejo inadequado da adubação reduz drasticamente a produtividade da cultura induzindo à reforma de canaviais em áreas que poderiam apresentar maior longevidade.

\section{CONCLUSÕES}

1) Reduções de doses e de nutrientes recomendados para a adubação mineral de soqueiras de cana-de-açúcar interferem significativamente no rendimento agrícola.

2) O retorno da adubação proporciona expressiva recuperação na produtividade das soqueiras não fertilizadas anteriormente.

3) O aumento da produtividade agrícola é significativo quando a adubação nitrogenada é a complementada com a potássica.

\section{AGRADECIMENTOS}

À administração da Usina Casquel pelas facilidades oferecidas para a execução deste trabalho.

\section{REFERÊNCIAS}

[1] AZEREDO, D. F.; MANHÃES, M. S.; ROBAINA, A. A. Adubação nitrogenada em cana-soca. SaccharumSTAB., São Paulo, v.3, n 9, p 35-38, 1980.

[2] AZEREDO, D. F.; ROBAINA, A. A.; ZANOTTI, N. E. Adubação mineral em cana-de-açúcar no Estado do Espírito Santo. Saccharum-STAB., São Paulo, v.7, n. 30, p 39-43, 1984.

[3] BOLSANELLO, J.; WEBER, H.; AZEREDO, D. F. Adubação fosfatada da cana-de-açúcar: doses e complementação. In: CONGRESSO NACIONAL DOS
TÉCNICOS ACUUCAREIROS E ALCOOLEIROS DO BRASIL, 5., 1993, Águas de São Pedro. Anais. Águas de São Pedro: STAB., 1993. p. 65-69.

[4] GOLDEN, L. E. Nutriente uptake by sugar cane in Louisiana. The Sugar Journal, New Orleans, v.23, n.11, p. 22-24. 1961

[5] HUMBERT, R. P. El cultivo de la caña de azucar. México: Editora Continental, 1974. 719 p.

[6] MARINHO, M. L. Aspectos agronômicos e econômicos da adubação da cana-de-açúcar em Alagoas. Rio Largo: EECA, 1974. 60 p.

[7] NUNES JÚNIOR, D. A redução da adubação e a produtividade. STAB, Piracicaba, v.17, n.3, p. 16, 1999.

[8] ORLANDO FILHO, J. Absorção dos macronutrientes pela cana-de-açúcar (Saccharum spp) variedade CB41-76, em três solos no Estado de São Paulo. Piracicaba, 1978. $154 \mathrm{f}$. Tese (Doutorado) Escola Superior de Agricultura Luiz de Queiroz / Universidade de São Paulo.

[9] RAIJ, B. van; CANTARELLA, H.; QUAGGIO, J. A.; FURLANI, A. M. C. Recomendação de adubação e calagem para o Estado de São Paulo. Campinas: IAC, 1996. (Boletim Técnico n.100 p 237-239).

[10]SANTOS, M. A. C.; SOBRAL, A. F.; CORDEIRO, D. A.; ARAÚJO, J. D. L. Adubação da cana-de-açúcar. Carpina: IAA/PLANALSUCAR, CONOR, 1973, 3 p. (Resumo informativo).

[11]SILVA, G. M. A.; ALONSO, O.; MORAIS, R. S. Influência da adubação sobre a produtividade e qualidade tecnológica da cana-de-açúcar. In: SEMINÁRIO COPERSUCAR DA AGROINDUSTRIA ACUCAREIRA, 4., 1977, Águas de Líndoia. Anais. São Paulo: Copersucar, 1977. p 27-35.

[12]WEBER, H.; AZEREDO, D. F. Adubação nitrogenada e potássica em diferentes ciclos de cana-soca e épocas durante a safra. In: SEMINÁRIO BIENAL DE PESQUISA DA UFRRJ, 8., 1997, Seropédica. Resumos. Seropédica: Imprensal Universitária, 1997. p. 28.

[13]ZAMBELLO JÚNIOR, E.; ORLANDO FILHO, J.; COLETTI, J. T.; ROSETTO, A. J. Adubação de soqueiras em três variedades de cana-de-açúcar (Saccharum spp) cultivadas em LE no Estado de São Paulo. Brasil Açucareiro, Rio de Janeiro, v.89, n.3, p. 11-17, 1977.

[14]ZAMBELLO JÚNIOR, E; AZEREDO, D. F. Adubação na Região Centro-Sul. In: ORLANDO FILHO, J. (Coord.) Nutrição e adubação da cana-de açúcar no Brasil. Piracicaba: IAA/PLANALSUCAR, SUPER, 1983. p. 287-313.

Recebido para publicação em 09 OUT 2000 [SA 031/2000] Aceito para publicação em 03 JUN 2002 